\title{
Physical Exercise and Gene p53-A Mini Review
}

\section{Tayane Horstmann Cabral ${ }^{1}$, Ana Luiza Lozinski' ${ }^{1}$, Gabriel Massao Tanaka1, Marco André Cardoso ${ }^{1}$, Rossana Baggio Simeoni ${ }^{2,3}$, Péricles Varella Gomes ${ }^{4}$, Zair Candido Oliveira Netto1, Luiz Cesar Guarita-Souza ${ }^{2}$, Julio Cesar Francisco ${ }^{1 *}$, Ricardo Correa Cunha1}

\author{
${ }^{1}$ School of Health Sciences, Positivo University, Curitiba, Brazil \\ ${ }^{2}$ School of Medical Sciences, Pontifical Catholic University of Paraná, Curitiba, Brazil \\ ${ }^{3}$ The Paraná Institute of Technology, Curitiba, Brazil \\ ${ }^{4}$ GEL LAB, Michigan State University East Lansing, East Lansing, USA \\ Email: ${ }^{*}$ julio.apfr@gmail.com
}

How to cite this paper: Cabral, T.H., Lozinski, A.L., Tanaka, G.M., Cardoso, M.A., Simeoni, R.B., Gomes, P.V., Netto, Z.C.O., Guarita-Souza, L.C., Francisco, J.C. and Cunha, R.C. (2020) Physical Exercise and Gene p53-A Mini Review. Journal of Cancer Therapy, 11, 463-469.

https://doi.org/10.4236/jct.2020.118039

Received: February 25, 2020

Accepted: August 15, 2020

Published: August 18, 2020

Copyright $\odot 2020$ by author(s) and Scientific Research Publishing Inc. This work is licensed under the Creative Commons Attribution International License (CC BY 4.0).

http://creativecommons.org/licenses/by/4.0/

\begin{abstract}
Cancer is a global problem that in addition to physical, emotional and physiological causes economic and social impacts. The p53 gene is a tumor suppressor gene found in many malignant and benign tumors; this has the primary function of keeping cells at rest after damaging to DNA. The p53 acts in the maintenance of cellular homeostasis, mainly through autophagy, playing a role in cell cycle arrest, when necessary, thus avoiding mutated DNA replication. When in the oncogenic environment in many cases it is mutated, losing much of its efficiency allowing tumor development. Studies show that exercise can in the regular part of its pro-autophagic function even in the oncology setting. Stimuli of moderate-intensity aerobic and predominance of submaximal seem to trigger the protective function of $\mathrm{p} 53$ in various cancer settings. Among the many changes that these pathology triggers were the objective of this mini review is to relate the changes that exercise generates in p53 protein functions and their possible influence on tumor cells.
\end{abstract}

\section{Keywords}

p53, Physical Exercise, Gene Mutations, Autophagy

\section{Introduction}

The tissues of the human body are formed by cells that multiply through a natural and orderly process; when this multiplication enters an entropy state, the diseases that have in common the disordered growth of cells are triggered, that is, a malignancy; malignant neoplasms are called cancer and benign neoplasms 
as tumors. Cancer consists of a change in genetic material and is characterized by progressive replacement of normal cells with altered cells, is among the main causes of morbidity and mortality worldwide and it is believed that in the next two decades the number of cases could increase by $70 \%$ [1].

Among the main causes of cancer are tobacco and alcohol consumption, an unhealthy diet and physical inactivity. Thus, making a major impact on the country, in this way measures are needed to minimize its effects on society. Among the alternatives aimed at preventing or treating cancer cases, physical exercise has been an intervention measure, together with conventional treatment, because it is an effective strategy, both in the prevention and treatment of patients' cancer [2].

The mechanisms involved in exercise can reduce tumor cells by increasing the transcription of p53 protein, and thus contribute to cancer prevention [3].

This function is tumor suppression and is known as "guardian of the genome", due to the fact that the TP53 gene is activated in response to signs of cellular damage, leading to transcription 1 of the p53 protein, which monitors the integrity of the genome and prevents the proliferation of cells with mutated DNA. Its transcription factor interacts with at least six other genes that are linked to the division of DNA and its processes [4] [5]. Based on this biological marker, the present study aimed to point out the relationship between physical exercise in the functions of the p53 protein and its influences on tumor cells.

\section{The Immune System and Cancer}

The main function of the immune system is to fight infections and toxic agents caused by pathogens. The genes considered tumor suppressors are essential to maintain genomic integrity and play an important role in the immune system, among them the p53 gene, associated with RB1, PTEN, and CDKN2, seem to respond to inflammatory diseases and signal immune responses [6]. These genes also amenable to mutation, can become oncogenes such as the case of TP53, exerting a dominant-negative effect, i.e., the only mutated product allele interacts with and inactivates the normal allele, inducing the processes cancer [5].

Tumor cells use several biological mechanisms for unable to proliferate, trying somehow not to be affected by the immune system. One such mechanism is the source of energy that they used, which is defined as a war burg effect and seems to facilitate the increase in cell mutated. Through accelerating excessive glycolysis and lactate formation, even in high availability of oxygen conditions, they cause changes in the environment cell phone. Lactogenesis that occurs in this process is associated with the chain respiratory cancer cells, thus resulting in high consumption of glucose and acid extracellular environment beyond normal levels [7].

Evidence shows that the P53 suppressor gene p53 and act in response to stress homeostasis state of the cell, since the change ribosomal to metabolic change, when in normal states, acting as an alert to the body, and when mutated contributes to cellular changes, increasing and decreasing glycolysis energy produc- 
tion, characteristic of respiration of tumor cells, thus attenuating the changes caused by cancer cells [7] [8].

Physical activities, mainly of moderate-intensity are known to improve the oxidative mitochondrial capacity and promoting activity of enzymes that are responsible for oxidation of glucose and acid fatty and may lead to a dispute by glucose, thereby decreasing availability supply to the tumor cells and the influence of mutated p53 gene, which plays an apparent role of enhancing the function of these cells [9].

\section{P53 and Possible Interactions with Exercise}

Exercise is usually associated with increased health and well being, nevertheless, a number of mechanisms by which it acts must be elucidated, for stimuli also represent a break constant in the body homeostasis, which before these various changes need to react, creating acute and chronic responses, especially in muscle tissue Skeletal. One way stimulated by exercise and coming gaining great prominence in the scientific field is the protein responses, these responses undergo several changes due to the entropic state caused in the cells by physiological changes occurring in the body due to different stimuli received [10].

Among the proteins that can be stimulated through exercise, those work in autophagy seem to have an important role in cancer because when autophagic activities are inhibited are afforded the genomes of re-productions unstable and chromosomal aberrations, then it is autophagy essential for genome stability and its inhibition is considered oncogenic [11] [12].

The p53 nuclear is a pro-autophagic, process triggered by transcription of several genes that are responsible for sensitivity to mTOR and AMPK while p53 cytoplasmic is suppressing autophagy, a process still not clear to researchers, but it may be associated with a negative regulation factor RB1 protein induced autophagy [12] [13].

This dual role is influential especially in scenarios cancer, as in healthy cells, even when p53 is found in cytoplasm, its interaction with other proteins and genes allows that there is apoptosis the non-functional cell since when treating the tumor cell, with mutated p53 undergo these interactions decreases, resulting in failure of cell cycle arrest, favoring a scenario where there is little propensity to apoptosis and autophagy, characteristics typical of cancer cells [5] [6].

Exercise seems to have a key role in this role p53, since several studies have shown that this protein reacts to stress caused by physical exercise, the data type of stimulation can increase or decrease its nuclear expression, mitochondrial and cytoplasmic, was also reported its ability to prevent or facilitate translocation of p53 to the nucleus mitochondria [14]. In an attempt to clarify some cases, studies of the area, selected for this systematic review, seek to work with different scenarios, such as the TP53 transmutation of studying cancer patients having the mutation, working with knocked out mice, cell handling "in vitro" [9].

The monitoring of the level of physical activity throughout the life of women diagnosis of breast cancer, mostly with mutations in TP53, showed a reduction 
in mortality risk associated with those practiced years Physical $1-10$ and $10-20$ years before diagnosis [9]. Corroborating these findings, experiments with rats, demonstrate that those exercised voluntarily (treadmill at 17.5 to $20 \mathrm{~m} / \mathrm{min}$ ), the period prepubescent, resulted in lower risk of development of triggers malignant transformation of breast tissue and improves the regulation of p53, while on sexual maturation, compared to the group of rats that did not exercise [14].

It shows how the exercise, done voluntarily and regularly, has the potential to generate a function protective interaction with the non-mutated p53 and prevent the possible activation triggers that could trigger the production of atypical cells in adulthood. In the presence of TP53 mutated, the findings are still controversial, similar stimuli, has led to different results [14].

\section{TP53 and the Mechanism for the Change Metabolic}

In the case of TP53 mutation, which results in the inactivation of p53 is one reported change in cell bioenergetics, it is more dependent on glycolysis and It has functionality drop in mitochondrial respiration, a process is known as Warburg effect, which could explain in part as a stimulus similar exercise results in different response in the presence or absence of mutated TP53 [9]. This decrease in mitochondrial function, previously reported, is confirmed in mice homozygotes, who also develop sarcomas and have a higher incidence of metastasis [15].

When comparing the homozygous mouse the mouse wild-type, there is a decrease in oxlylgen consumption homozygous species, related to p53 deficiency, even if the final amount to amount of ATP is similar in all genotypes, those with disabilities genetics, produced high levels of lactate, confirming the energy cell change, that favors glycolysis and low aerobic capacity [16]. The regulation of aerobic respiration by $\mathrm{p} 53$, corroborates the fact that your inactivation may promote tumorigenesis by decreasing the dependence oxygen would result in more and more acidotic hypoxic environments, furthermore the loss of p53 function may undo a cellular response that is exerted on the excessive presence of reactive oxygen species [9] [16].

Hypoxia is one of the most important flags for angiogenesis in tumors, vasculature that is known to form a network that strengthens the cell tumor and its processes. The cellular response to hypoxia is given largely by HIF- $1 \alpha$, the main transcriptional regulator of cellular response and development to hypoxia. The HIF- $1 \alpha$ responds to the low presence of oxygen and activates the transcription genes resulting in glycolytic enzymes, glucose and lactate transporter, which is also responsible for the transcription of the gene MCT4, which favors the extrusion lactate cell [17].

This availability of lactate can do with which it is absorbed by other cells, it can be used in aerobic metabolism or converted back into glucose in the liver, potentially leading to energy waste and cachexia. Lactate which is highly influenced HIF-1 is considered a key component of the metabolic Warburg effect, as you may be involved in all processes of carcinogenesis, such as signaling for proliferation, cancellation of the suppressive effects of growth, and metastasis 
mainly in angiogenesis. Strenuous exercise can lead to hypoxia, and they are related to hormones and cytokines, types of modulation Reactive oxygen, and DNA damage [18].

The mechanical damage that may be caused by these exercises results in apoptosis and necrosis with inflammatory responses, increases also the production of reactive oxygen species, studying animals exposed to the training and hypoxia chamber led to increased p53 and TNF- $\alpha$ leading to increased apoptosis of healthy lung tissue. Due to the naturally hypoxic cells in the framework of a situation where the TP53 gene is mutated, the use of strenuous exercise that leads to an enhancement of more acidotic and lactogenic environment, can result in the table marked with TNF- $\alpha$ expression, so that there is signaling apoptosis of healthy cells, in a disorderly manner [19] [20].

\section{P53 in Acute Responses to Exercise}

Regarding the acute responses to exercise, we have different results in the ratio p53 location. In an experiment where men were untrained subjected to cycle ergometer exercise for 60 minutes $\left(70 \%\right.$ of peak $\left.\mathrm{VO}^{2}\right)$ It resulted in increased nuclear p53, but did not suggest changes in mitochondrial p53, with results similar study in transgenic mice, subjected to 60-minute submaximal exercise (treadmill for Run $12-18 \mathrm{~m} / \mathrm{min}$ ) [21] [22].

Already in rats on a treadmill for 90 minutes $(15 \mathrm{~m} / \mathrm{min})$, there was the decrease of nuclear p53 and p53 signaling increase mitochondrial rats wild-type, in rats and knocked out, adaptations to exercise It was zero in relation to proteins related to p53. THE results of difference may be related to different protocols, species, or Indeed human and the mutated are untrained rats specific proteins [21] [22] [23].

\section{Final Conclusions and Future Perspectives}

Cells can interact with variables of physical exercise and in this case more specifically p53. Exercise at moderate intensity and submaximal aerobic predominance seems to trigger protective functions p53 in various cancer settings, primarily through stimulating cell autophagy.

High-intensity exercise triggers the hypoxia and increases lactic acid and angiogenesis. These factors, besides the action of HIF and the suppression of p53 protein, are considered protective factors to the vascular network of tumor cells.

Still results of differences exist, because as in any intervention involving oncology, the protocols do not apply to all patients and all types of tumor cells, as in any intervention involving oncology, the protocols do not apply to all patients and types of tumor cells. However, some findings demonstrate the benefits of exercise when compared to the group without exercise in animals. The p53 and physical exercise have a notable role and are still controversial in cancer scenarios, but they appear to be interesting tools for the quality of life and as an alternative treatment for some tumorigenic scenarios. 


\section{Conflicts of Interest}

The authors declare no conflicts of interest regarding the publication of this paper.

\section{References}

[1] Global Burden of Disease Cancer C., et al. (2018) Global, Regional, and National Cancer Incidence, Mortality, Years of Life Lost, Years Lived with Disability, and Disability-Adjusted Life-Years for 29 Cancer Groups, 1990 to 2016: A Systematic Analysis for the Global Burden of Disease Study. JAMA Oncology, 4, 1553-1568. https://doi.org/10.1200/JCO.2018.36.15_suppl.1568

[2] Jiang, H., et al. (2019) Can Public Health Policies on Alcohol and Tobacco Reduce a Cancer Epidemic? Australia's Experience. BMC Medicine, 17, Article No. 213. https://doi.org/10.1186/s12916-019-1453-Z

[3] Yu, M., et al. (2016) Exercise Activates p53 and Negatively Regulates IGF-1 Pathway in Epidermis within a Skin Cancer Model. PLoS ONE, 11, e0160939. https://doi.org/10.1371/journal.pone.0160939

[4] Leroy, B., et al. (2014) Analysis of TP53 Mutation Status in Human Cancer Cell Lines: A Reassessment. Human Mutation, 35, 756-765. https://doi.org/10.1002/humu.22556

[5] Mantovani, F., Collavin, L. and Del Sal, G. (2019) Mutant p53 as a Guardian of the Cancer Cell. Cell Death \& Differentiation, 26, 199-212. https://doi.org/10.1038/s41418-018-0246-9

[6] Kastenhuber, E.R. and Lowe, S.W. (2017) Putting p53 in Context. Cell, 170, 1062-1078. https://doi.org/10.1016/j.cell.2017.08.028

[7] Eriksson, M., et al. (2017) Effect of Mutant p53 Proteins on Glycolysis and Mitochondrial Metabolism. Molecular and Cellular Biology, 37, e00328-17. https://doi.org/10.1128/MCB.00328-17

[8] Napoli, M. and Flores, E.R. (2017) The p53 Family Orchestrates the Regulation of Metabolism: Physiological Regulation and Implications for Cancer Therapy. British Journal of Cancer, 116, 149-155. https://doi.org/10.1038/bjc.2016.384

[9] Tao, M.H., et al. (2013) Association of Prediagnostic Physical Activity with Survival Following Breast Cancer Diagnosis: Influence of TP53 Mutation Status. Cancer Causes Control, 24, 2177-2186. https://doi.org/10.1007/s10552-013-0294-X

[10] Meneses-Echavez, J.F., Gonzalez-Jimenez, E. and Ramirez-Velez, R. (2015) Effects of Supervised Multimodal Exercise Interventions on Cancer-Related Fatigue: Systematic Review and Meta-Analysis of Randomized Controlled Trials. BioMed Research International, 2015, Article ID: 328636. https://doi.org/10.1155/2015/328636

[11] Helma, R., et al. (2019) p53 Binds Preferentially to Non-B DNA Structures Formed by the Pyrimidine-Rich Strands of GAA TTC Trinucleotide Repeats Associated with Friedreich's Ataxia. Molecules, 24, 2078. https://doi.org/10.3390/molecules24112078

[12] Nassour, J., et al. (2019) Autophagic Cell Death Restricts Chromosomal Instability during Replicative Crisis. Nature, 565, 659-663. https://doi.org/10.1038/s41586-019-0885-0

[13] Silva, J.V., et al. (2019) mTOR Signaling Pathway Regulates Sperm Quality in Older Men. Cells, 8, 629. https://doi.org/10.3390/cells8060629 
[14] Wang, M., et al. (2009) Prepubertal Physical Activity Up-Regulates Estrogen Receptor Beta, BRCA1 and p53 mRNA Expression in the Rat Mammary Gland. Breast Cancer Research and Treatment, 115, 213-220. https://doi.org/10.1007/s10549-008-0062-x

[15] van Boxtel, R., et al. (2011) Homozygous and Heterozygous p53 Knockout Rats Develop Metastasizing Sarcomas with High Frequency. The American Journal of Pathology, 179, 1616-1622. https://doi.org/10.1016/j.ajpath.2011.06.036

[16] Matoba, S., et al. (2006) p53 Regulates Mitochondrial Respiration. Science, 312, 1650-1653. https://doi.org/10.1126/science.1126863

[17] San-Millan, I. and Brooks, G.A. (2017) Reexamining Cancer Metabolism: Lactate Production for Carcinogenesis Could Be the Purpose and Explanation of the Warburg Effect. Carcinogenesis, 38, 119-133. https://doi.org/10.1093/carcin/bgw127

[18] Huang, Y., Lin, D. and Taniguchi, C.M. (2017) Hypoxia Inducible Factor (HIF) in the Tumor Microenvironment: Friend or Foe? Science China Life Sciences, 60, 1114-1124. https://doi.org/10.1007/s11427-017-9178-y

[19] Wigerup, C., Pahlman, S. and Bexell, D. (2016) Therapeutic Targeting of Hypoxia and Hypoxia-Inducible Factors in Cancer. Pharmacology \& Therapeutics, 164, 152-169. https://doi.org/10.1016/j.pharmthera.2016.04.009

[20] Pentimalli, F., et al. (2019) Cell Death Pathologies: Targeting Death Pathways and the Immune System for Cancer Therapy. Genes \& Immunity, 20, 539-554.

https://doi.org/10.1038/s41435-018-0052-x

[21] Zhuang, J., et al. (2016) Forkhead Box O3A (FOXO3) and the Mitochondrial Disulfide Relay Carrier (CHCHD4) Regulate p53 Protein Nuclear Activity in Response to Exercise. The Journal of Biological Chemistry, 291, 24819-24827. https://doi.org/10.1074/jbc.M116.745737

[22] Tachtsis, B., et al. (2016) Acute Endurance Exercise Induces Nuclear p53 Abundance in Human Skeletal Muscle. Frontiers in Physiology, 7, 144. https://doi.org/10.3389/fphys.2016.00144

[23] Saleem, A. and Hood, D.A. (2013) Acute Exercise Induces Tumour Suppressor Protein p53 Translocation to the Mitochondria and Promotes a p53-Tfam-mitochondrial DNA Complex in Skeletal Muscle. Journal of Physiology, 591, 3625-3636.

https://doi.org/10.1113/jphysiol.2013.252791 